\title{
Improving Specificity of Breast MRI Using Prone PET and Fused MRI and PET 3D Volume Datasets
}

Linda Moy ${ }^{1}$, Fabio Ponzo ${ }^{1}$, Marilyn E. Noz ${ }^{1}$, Gerald Q. Maguire, Jr. ${ }^{2}$, Antoinette D. Murphy-Walcott ${ }^{1}$, Abby E. Deans ${ }^{1}$, Mary T. Kitazono ${ }^{1}$, Laura Travascio ${ }^{1}$, and Elissa L. Kramer ${ }^{1}$

${ }^{1}$ Department of Radiology, School of Medicine, New York University, New York, New York; and ${ }^{2}$ School of Information and Communication Technology, Royal Institute of Technology, Kista, Sweden

$M R I$ is a sensitive method for detecting invasive breast cancer, but it lacks specificity. To examine the effect of combining PET with MRI on breast lesion characterization, a prototype positioning device was fabricated to allow PET scans to be acquired in the same position as MRI scans_that is, prone. Methods: To test the hypothesis that fusion of ${ }^{18} \mathrm{~F}$-FDG PET and MRI scans improves detection of breast cancer, 23 patients with suspected recurrent or new breast cancer underwent a routine whole-body PET scan, a prone PET scan of the chest, and a routine breast MRI scan. The attenuation-corrected prone PET and MRI datasets were registered twice by different operators. The fusion results were judged for quality by visual inspection and statistical analysis. A joint reading of the MRI and PET scans side by side and integrated images was performed by a nuclear medicine physician and a radiologist. Sensitivity and specificity of MRI and combined MRI and PET scans were calculated on the basis of pathology reports or at least $1 \mathrm{y}$ of clinical and radiologic follow-up. Results: All fusions were verified to be well matched using specific anatomic criteria. A total of 45 lesions was assessed. Lesion size range was 0.6 to $10.0 \mathrm{~cm}$. Of the 44 breasts examined, 29 were suspicious for cancer, of which 15 were found to be positive on surgical excision. In lesion-by-lesion analysis, sensitivity and specificity of MRI alone were $92 \%$ and $52 \%$, respectively; after MRI and PET fusion, they were $63 \%$ and $95 \%$, respectively. The positive predictive value and the negative predictive value for MRI alone were $69 \%$ and $85 \%$, respectively; after MRI and PET fusion, they were $94 \%$ and $69 \%$, respectively. Conclusion: Acquisition of prone PET scans using the new positioning device permitted acquisition of prone scans suitable for fusion with breast MRI scans. Fused PET and MRI scans increased the specificity of MRI but decreased the sensitivity in this small group of patients. Additional data are needed to confirm the statistical significance of these preliminary findings.

Key Words: MRI and PET fusion; breast cancer; specificity; prone PET device; prone PET breast scans

J Nucl Med 2007; 48:528-537

DOI: 10.2967/jnumed.106.036780

Received Sep. 26, 2006; revision accepted Dec. 12, 2006.

For correspondence or reprints contact: Marilyn E. Noz, PhD, School of Medicine, New York University, 550 First Ave., New York, NY 10016.

E-mail: marilyn.noz@med.nyu.edu

COPYRIGHT @ 2007 by the Society of Nuclear Medicine, Inc.
$\mathbf{P}$ rior studies have demonstrated that breast MRI may be used to detect breast cancers that are occult on mammography, sonography, and clinical breast examination (1-3). MRI has a high sensitivity for identifying tissue abnormalities, but the reported specificities are variable $(4,5)$. MRI breast examinations are performed with the patient in the prone position because the breasts are flattened and suboptimally visualized in the supine position. The value of comparing upper-body ${ }^{18} \mathrm{~F}-\mathrm{FDG}$ PET scans with MRI scans to detect axillary and supraclavicular tumors has been examined (6). It has been shown (7) that the higher standardized uptake values (SUVs) provided by prone ${ }^{18} \mathrm{~F}-\mathrm{FDG}$ PET breast imaging in cancer detection were significantly different from those obtained in supine imaging alone and increased the confidence of the readers. The value of using prone PET with MRI scans has been examined, simply using side-by-side comparison (8).

Integrated PET and CT (PET/CT) machines, enabling serial acquisition and subsequent display as a single fused image, are commercially available and, for breast imaging, have shown some improvement over PET alone (9). A comparable system that could acquire and fuse MRI and PET scans is not currently commercially available, though there are some experimental units that acquire the 2 scans simultaneously (10) and clinical units are under development.

Our hypothesis is that by fusion of the metabolic information obtained by ${ }^{18}$ F-FDG PET scans with the MRI scan, the specificity of enhancing masses detected on MRI can be increased. To facilitate fusion of PET and MRI studies, a prototype positioning device, shown in Figure 1, was designed to allow the patient to be imaged in a prone position, with breasts pendant, hanging freely under gravity. This permitted better visualization of the breast tissue using a similar physical configuration to MRI (11). The increased specificity of MRI might then spare the patient the trauma of more invasive procedures (i.e., fine-needle aspiration [FNA], biopsy, or resection) or the anxiety of a series of short-interval follow-up examinations. 


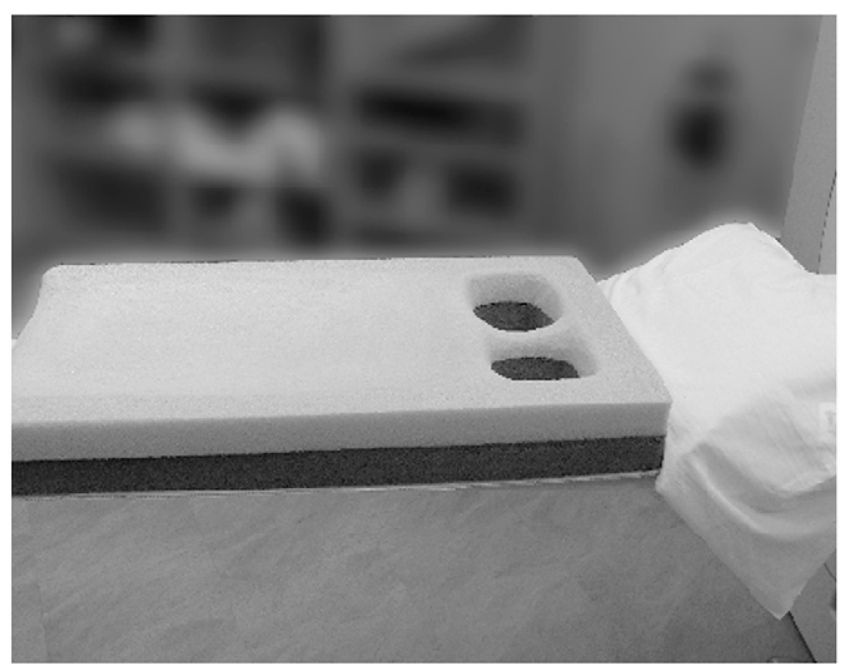

FIGURE 1. Breast apparatus used to position patient for prone ${ }^{18} \mathrm{~F}-\mathrm{FDG}$ PET study is shown positioned on scanner couch. Patient's shoulders, arms, and head rest comfortably on pillows provided.

\section{MATERIALS AND METHODS}

\section{Patients}

Between January 2005 and January 2006, 23 women were examined with ${ }^{18}$ F-FDG PET and MRI scans because of suspected recurrent disease or suspected multifocal, multicentric, or contralateral disease. Consecutive patients were recruited for additional evaluation with PET/CT or breast MRI if either study detected an abnormality. An attempt was made to schedule both studies as temporally close as possible. All patients had a routine breast MRI scan within 4 mo (scanning range, 0-111 d; mean, $19 \mathrm{~d}$ ) of the PET scan. However, for 7 of the patients, the PET scan was done as a precautionary follow-up; thus, the interval between the PET and MRI scan was longer (19-111 d). Most patients had a routine PET scan within $0-5 \mathrm{~d}$ of the MRI scan. Although the patients ranged in age from 24 to $65 \mathrm{y}$ (mean age, $41 \mathrm{y}$ ), 16 patients were $<50 \mathrm{y}$ of age.

The physician read the original studies side by side if possible, as both examinations were part of the patient's standard of care. The fusion study was approved by the New York University School of Medicine Institutional Review Board and informed consent was obtained from each patient.

\section{Reference Standard}

Surgical excision or core biopsy/cytology results were used as a reference standard. If not available, patients with negative findings on MRI or negative PET findings underwent follow-up examinations for at least $1 \mathrm{y}$ (range, 12-20 mo) using additional imaging, such as breast MRI, mammography, ultrasound (US), and clinical evaluation.

\section{PET/CT Data Volumes}

Datasets were acquired using a combined PET/CT in-line system (HiRes pico/Biograph 6; Siemens Medical Solutions USA, Inc.). This is a high-resolution lutetium oxyorthosilicate crystal PET scanner integrated with a 6-detector CT scanner. The PET component uses a 3-dimensional (3D) acquisition technique and includes an axial field of view of $15.5 \mathrm{~cm}$ and a transaxial resolution of $4.2 \mathrm{~mm}$ at $1 \mathrm{~cm}$ from the center.

Patients fasted at least $6 \mathrm{~h}$ before injection of $370-740 \mathrm{MBq}$ of ${ }^{18}$ F-FDG based on the patient's weight. Scanning was started
$45 \mathrm{~min}$ after injection. Blood glucose levels were $<150 \mathrm{mg} / \mathrm{dL}$ ( $8.3 \mathrm{mmol} / \mathrm{L})$. No intravenous contrast agent was administered. Initially, patients were examined in the supine position with arms elevated, and CT was started at the level of the base of the skull with the following parameters: $80 \mathrm{mAs}$; 140-kVp CARE Dose4D, which automatically adjusts the CT tube current to minimize the radiation dose to the patient while maintaining image quality; slice spacing, 2.5 $\mathrm{mm}$; pitch, 1.5. The CT scans were acquired during shallow breathing and reached caudally to the midthigh. PET over the same region was performed immediately after acquisition of the CT images.

Approximately $1 \mathrm{~h} 10 \mathrm{~min}$ after ${ }^{18} \mathrm{~F}-\mathrm{FDG}$ injection, a second CT data acquisition set from the base of the neck to the upper abdominal region was initiated in the prone position for a total of 2 bed positions. The CT acquisition had the same parameters as before for the supine position, except that in the first 9 cases the slice spacing was $2.5 \mathrm{~mm}(n=9)$ and subsequently was changed to $4.0 \mathrm{~mm}(n=13)$ to improve the attenuation correction and decrease study time. A prone PET dataset over the same region was acquired immediately after acquisition of the CT images.

For prone patient positioning, a new positioning device (Fig. 1) was used, allowing the same patient geometry as the commercial breast MRI coil. This permitted the PET scan to be similar to the MRI breast configuration. CT data were used for attenuation correction, and the PET scan was reconstructed (applying a standard iterative algorithm [ordered-subset expectation maximization]) into a $168 \times 168$ matrix; the number of slices ranged from 70 to 157 for the prone studies, depending on the slice spacing used for the attenuation-correction CT. The $x-y$ pixel size was $4.06 \mathrm{~mm}$ and the slice thickness was 2.5 or $4.0 \mathrm{~mm}$, depending on the slice spacing used in the attenuation-correction CT.

\section{Diagnostic Breast MRI Technique and MRI Data Volumes}

The breast MRI examinations were performed with the patient prone in a 1.5-T commercial imager (Sonata, Symphony, or Avanto; Siemens Medical Solutions) $(n=19)$ or a 3-T commercial imager (TIM Trio; Siemens Medical Solutions) with the use of a dedicated surface breast coil (MRI Devices). The imaging sequence includes a sagittal T1-weighted localizing sequence followed by a sagittal T2weighted sequence $(8,990 / 107$ [repetition time (TR)/echo time (TE)]). A T1-weighted 3D, fat-suppressed fast spoiled-gradientecho sequence (TR/TE, 8.89/1.7; flip angle, $12^{\circ}$; bandwidth, 34.5 $\mathrm{Hz}$ ) was then performed before and 4 times after a rapid bolus injection of $0.1 \mathrm{mmol}$ per kilogram body weight of gadolinium dimeglumine (Magnevist; Schering) at an injection rate of $2.0 \mathrm{~mL} / \mathrm{s}$. The protocol for the 3.0-T imager was similar to that of the $1.5-\mathrm{T}$ imager (Table 1).

TABLE 1

Breast MRI Parameters

\begin{tabular}{lcl}
\hline \multicolumn{1}{c}{ Parameter } & 1.5-T unit & 3.0-T unit \\
\hline T2 imaging TR/TE & 8,990/107 & 8,400/103 \\
T1 imaging & 3D T1 Fat-sat & 3D T1 Fat-sat \\
& FLASH & FLASH \\
T1 imaging TR/TE & $8.89 / 1.7$ & $7.8 / 2.9$ \\
Flip angle (degrees) & 12 & 8 \\
Bandwidth (Hz) & 34.5 & 71.0
\end{tabular}

Fat-sat FLASH $=$ fat-saturation fast low-angle shot. 
Image acquisition began immediately after administration of the contrast material and saline bolus. Total examination time per breast, including the 4 examinations that were performed after the administration of contrast material, was approximately $15 \mathrm{~min}$.

An axially reconstructed $3 \mathrm{D}$ breath-hold fat-saturation sequence was available for 21 of the 23 patients. When no axially reconstructed volumes were available, locally developed software was used to reorient the volumes into the axial plane, which reduced the resolution in the $x$-direction from $0.59 \mathrm{~mm}$ in one case and $0.52 \mathrm{~mm}$ in the other, to $1.6 \mathrm{~mm}$. The MRI volumes were reconstructed into various matrix sizes that ranged in $x$ from 160 to 512 pixels, in $y$ from 320 to 512 pixels, and in $z$ from 29 to 384 slices. The pixel sizes ranged in $x$ from 0.625 to $1.6 \mathrm{~mm}$ and in $y$ from 0.52 to $1.09 \mathrm{~mm}$; the slice spacing, $z$, ranged from 0.52 to $6.0 \mathrm{~mm}$.

\section{Image Analysis}

Both PET/CT and breast MRI were performed as an extent of disease evaluation in all of the patients. The breast MR images were evaluated prospectively according to the American College of Radiology (ACR) BIRADS (Breast Imaging Reporting and Data System) lexicon as part of the patients' routine clinical workup. Abnormal enhancement was characterized as mass or nonmass lesions. Both the morphologic appearance (size, shape, and pattern of enhancement) and the temporal enhancement pattern were evaluated. Time signal intensity curves (progressive, plateau, or washout) were generated for all enhancing lesions. The level of suspicion was reported on a scale of $1-6$, per the ACR BIRADS lexicon, with $1=$ no abnormal enhancement; $2=$ benign; 3 = probably benign, short-interval follow-up (usually in $6 \mathrm{mo}$ ); 4 = suspicious for malignancy; $5=$ highly suggestive of malignancy; and $6=$ known malignancy.

${ }^{18}$ F-FDG PET scans were reviewed first by inspection of a maximum-intensity-projection image followed by visualization of fused PET/CT slices in the standard orthogonal (coronal, transaxial, and sagittal) planes. CT was used for anatomic localization.

Findings were categorized as positive or negative for malignancy. Lesions within tissues of mild metabolic activity were classified as positive when ${ }^{18} \mathrm{~F}$-FDG uptake was greater than blood-pool activity. Lesions within tissues demonstrating moderate or high physiologic activity (such as liver) were considered positive if the activity was greater than the adjacent physiologic activity. Additionally, semiquantitative analysis was attempted using SUVs. Nine by 9 pixel regions of interest (ROIs) were chosen over all areas of abnormal ${ }^{18}$ F-FDG uptake in the suspicious breast. When no lesions were seen on CT or no abnormal ${ }^{18}$ F-FDG uptake was seen on PET images, then ROIs were placed in the region of the abnormalities indicated by the MRI. Their location was determined by using the MRI to approximate the location on the coregistered CT scan. In each ROI, the maximum SUV corrected for body weight was calculated. If possible, SUVs were also obtained in the contralateral breast in the same region where a focal abnormality was seen; otherwise, SUVs were obtained in a region that was closest in tissue density.

\section{PET and MRI Fusion}

Once the side-by-side analysis was completed for clinical purposes, the PET and MRI data were fused. In this preliminary study, a semiautomated (12) fully nonaffine fusion method was used. This allows nonrigid warping of image data to facilitate fusion even when some soft-tissue distortion is present, as it was not possible to treat these volumes as rigid bodies.
The ${ }^{18} \mathrm{~F}-\mathrm{FDG}$ PET/CT and MRI scans were transferred in DICOM (Digital Imaging and Communication in Medicine) format via the hospital network to workstations (Sun ULTRA10, Elite 3D6m; Sun Microsystems) in the nuclear medicine facility. After the data were reformatted for the volume fusion program (codeveloped with Saya Systems Inc.), 3D volume registration was accomplished using a previously described and validated fusion program (13-15).

The attenuation-corrected PET and MRI data were fused by 3 operators who were unaware of the results. All operators participated in landmark picking and the initial fusion evaluation task. Operator 1 was an experienced nuclear medicine technologist who had extensive experience with this process; operator 2 was a fourth year medical student, and operator 3 was an $\mathrm{MD} / \mathrm{PhD}$ student. Neither of latter 2 operators had any previous nuclear medicine image experience.

The PET scan was treated as the volume to be registered-that is, the floating volume-and the MRI scan was the reference volume, although the software supports either choice. Cohomologous points, commonly called landmarks, were chosen on concurrently viewed slices that display the same physiologic site or structure. Once a landmark was chosen, the pixel was marked and a sequential number was assigned. Landmarks were chosen to be as widely distributed as possible throughout the volume. Once a user is familiar with the method, these landmarks can be picked rather rapidly. In this specific application, a minimum of 12 absolute or relative landmarks was required; in most cases, between 20 and 40 landmarks were placed. Absolute landmarks were placed on recognizable anatomic structures-for example, on a specific part of the nipple. Relative landmarks, defined as anatomic structures/points relative to an absolute landmark-for example, matching the lungs by comparing which lobes of the lung were imaged-were also used. Matching the heart to the level that was imaged - for example, at the level of the apex of the left ventricle-was also done. Although skin boundaries are generally hard to determine on radionuclidic images, the outline of the breast was used when possible.

The paired landmarks were used as input parameters to obtain the transformation coefficients for a warping transformation. Each voxel in the floating volume was transformed into correspondence with the reference image. To facilitate evaluation of the fusion, the transformed floating volume was shown as 2-dimensional (2D) slices at the same anatomic level as the reference volume. The 2D renderings may be viewed side by side or merged with the reference slices. The volumes may also be displayed in 3D either separately or superimposed with an isosurface from another volume for comparison. The 3D volumes can be rotated and viewed from an arbitrary direction. The landmarks can be chosen or viewed on this 3D display (13-15).

This fusion method takes about 10-30 min (depending on the skill of the user) for viewing the slices, selecting landmarks, and generating a satisfactory new, registered, volume dataset for each patient.

\section{Data Analysis}

Although PET and MRI datasets for each patient were registered twice, visual inspection of the fusion was performed first by the operators and then by several participants in this project, using anatomic criteria that included similar size and contour of the breast at each slice, as well as good overlap of the costophrenic angles, great vessels, and heart. ANOVA on unequal sample sizes was applied to the fusion results. 
After fusion, 2 "blinded" physicians (specializing in breast imaging and nuclear medicine) retrospectively reread both studies. During the readout session, both readers reviewed the PET and MR images side by side. Afterward, both readers reviewed the superimposed fused images performed by only the first operator. These results were compared with individual clinical reports of the MRI and PET scans before fusion on a site-by-site basis. Sites identified in the original PET and MRI reports were reevaluated. If fusion identified additional foci of suspicious activity, the sites of the foci were logged.

In a separate readout session, 1 month later, both readers again reviewed the PET and MRI data side by side. Afterward, they reviewed the fused images performed by the second and third operators.

Using the histology/cytology reports or radiologic follow-up of at least $1 \mathrm{y}$, the numbers of true-negative (TN), true-positive (TP), false-negative (FN), and false-positive (FP) results were obtained for MRI alone and for fused MRI and PET. A McNemar test was applied to the MRI alone and PET and MRI positive and negative results to determine statistical independence.

Sensitivity and specificity as well as positive predictive value (PPV) and negative predictive value (NPV) of MRI alone and fused MRI and PET scans were calculated from the data.

\section{RESULTS}

\section{Patient Population}

Twenty-two of 23 patients' scans were available for fusion: 1 patient was excluded because the PET scan was acquired during chemotherapy. A total of 44 breasts were imaged, but only the 29 breasts with suspicious lesions on MRI were included in this study, yielding a total of 45 lesions for analysis.

\section{Evaluation of Fusion Technique}

For the 22 clinical cases, each fusion (44 fusions, each performed by 1 of the 3 different operators) was judged to be visually acceptable by the previously stated anatomic criteria. ANOVA on unequal sample sizes showed that the registration methodology between operators and across patients was not significantly different $(P>0.05)$.

\section{Histology/Cytology and Radiologic Follow-up}

Of the 45 lesions assessed, 7 were seen in the contralateral breast. As measured from MRI, the lesions ranged in size from 0.6 to $10.0 \mathrm{~cm}$ (mean, $2.5 \mathrm{~cm}$ ). Of the 44 breasts examined, 29 were suspicious for cancer, based on the MRI examination. Cancer was present in 22 breasts and absent in 7. Mastectomy was performed on 13 breasts, lumpectomy or surgical excision on 5 breasts, and core biopsy $(n=1)$ or FNA $(n=2)$ on 3 breasts. For the remaining 8 breasts, clinical and radiologic follow-up of at least $1 \mathrm{y}$ was performed on 11 lesions (Table 2).

Breast cancer was not present in any of 7 cases with equivocal findings in the contralateral breast. In these cases, 4 lesions had histologic correlation (mastectomy in 3 patients, core biopsy in 1 patient); and 3 subjects underwent follow-up for at least $1 \mathrm{y}$. Table 3 gives a lesion-by-lesion analysis of each MRI finding and the PET data correlated with it. In
TABLE 2

Pathologic or Radiologic Follow-up of Breast Lesions

\begin{tabular}{lcc}
\hline & $\begin{array}{c}\text { No. of } \\
\text { patients }\end{array}$ & $\begin{array}{c}\text { No. of } \\
\text { breasts }\end{array}$ \\
\hline Mastectomy & 10 & 13 \\
Surgical excision or lumpectomy & 5 & 5 \\
Core biopsy/FNA & 2 & 3 \\
Radiologic follow-up & 5 & 8 \\
\hline
\end{tabular}

summary, 1 lesion was classified as BIRADS 1,15 as BIRADS 3,12 as BIRADS 4, 3 as BIRADS 5, and 14 as BIRADS 6.

\section{Evaluation of MRI Data and MRI Data Fused with Hypermetabolic Activity in Breast}

In the lesion-by-lesion analysis for the 45 lesions, MRI alone was TP in 22 lesions (49\%), TN in 11 lesions (24\%), FN in 2 lesions (4.4\%), and FP in 10 lesions (22\%). Fused MRI and PET was TP in 14 lesions (33\%), TN in 20 lesions (44\%), FN in 5 lesions (11\%), and FP in 1 lesion (2\%). Fused MRI and PET was helpful in correctly characterizing 34 lesions (76\%) but incorrectly identified 1 "abnormality" not initially noted on MRI or PET alone. Table 4 summarizes the TP, TN, FP, and FN results for the 45 lesions on MRI and fused MRI and PET. A McNemar test showed that the results from MRI and from the fused MRI and PET were statistically independent $(P<0.05)$.

In the lesion-by-lesion analysis for the 45 lesions in the primary breast, sensitivity and specificity of MRI were $92 \%$ and 52\% and for fused MRI and PET were equal to 63\% and 95\%, respectively. The PPV and NPV for MRI alone was $69 \%$ and $85 \%$ and for fused MRI and PET was $94 \%$ and $69 \%$, respectively. Table 4 summarizes the results for the sensitivity, specificity, PPV, and NPV for the 45 lesions.

There were no lesions that demonstrated increased metabolic activity on PET alone that was not seen on MRI. However, during 1 of 2 readout sessions, 1 FP lesion was seen on the PET study.

The SUVs were grouped into 3 categories: those below which all lesions were proven negative (range, 0.0-0.79), those above which all lesions were proven positive (above 2.96), and those in which the SUVs (range, 0.8-2.95) overlapped. In the overlap range, some lesions were proven positive $(n=7)$ and some lesions were proven negative $(n=15)$. Using a 2-sample, nonpaired, Student $t$ test, the overlapping SUVs were found to be not significantly different $(P=0.66)$. Additionally, a Student $t$ test applied to the SUVs for the contralateral breast was shown to be not significantly different $(P>0.5)$ from an SUV of 0.6. A density plot of the contralateral SUVs showed them to be normally distributed about a mean of 0.6 , which is within the range of negative SUVs.

\section{Case Examples}

The results are further illustrated by considering the findings for 3 typical patients. Patient 1, a 25-y-old woman, 
TABLE 3

Patient Findings

\begin{tabular}{|c|c|c|c|c|c|c|c|c|c|}
\hline Patient & Lesion & $\begin{array}{l}\text { Size } \\
(\mathrm{cm})\end{array}$ & $\begin{array}{c}\mathrm{MRI} \\
\text { finding }\end{array}$ & $\begin{array}{c}\text { BIRADS } \\
\text { assessment }\end{array}$ & $\begin{array}{l}\text { PET/CT } \\
\text { finding } \\
\text { maximum } \\
\text { SUV }\end{array}$ & $\begin{array}{c}\text { Contralateral } \\
\text { breast }^{\dagger}\end{array}$ & $\begin{array}{l}\text { Normal SUV } \\
\text { contralateral } \\
\text { breast }^{\ddagger}\end{array}$ & Pathology & $\begin{array}{l}\text { Follow-up } \\
\text { or histology }\end{array}$ \\
\hline 1 & 1 & 3.3 & L mass & 3 & 1.53 & No & $\mathrm{R}, 0.4$ & $\mathrm{~N} / \mathrm{A}$ & $20 \mathrm{mo}$ \\
\hline 2 & 1 & 2.0 & L mass & 3 & 1.34 & No & $\mathrm{R}, 0.17$ & $\mathrm{~N} / \mathrm{A}$ & $15 \mathrm{mo}$ \\
\hline 2 & 2 & 1.7 & L mass & 3 & 1.22 & No & $R, 0.39$ & $\mathrm{~N} / \mathrm{A}$ & $15 \mathrm{mo}$ \\
\hline 3 & 1 & 3.0 & $\mathrm{R}$ mass & 6 & 2.6 & No & $L, 0.46$ & $\mathrm{~N} / \mathrm{A}$ & $19 \mathrm{mo}$ \\
\hline 4 & 1 & 2.0 & L mass & 3 & 1.82 & No & $R, 0.22$ & $\mathrm{~N} / \mathrm{A}$ & $13 \mathrm{mo}$ \\
\hline 5 & 1 & 0.8 & $\mathrm{R}$ mass & 3 & 1.34 & No & $L, 0.28$ & $\mathrm{~N} / \mathrm{A}$ & $19 \mathrm{mo}$ \\
\hline 6 & 1 & 0.7 & $\mathrm{R}$ mass & 4 & 0.4 & No & $\begin{array}{l}\mathrm{L}, \text { no breast } \mathrm{s} / \mathrm{p} \\
\text { mastectomy }\end{array}$ & Fibrocystic change & US core biopsy \\
\hline 6 & 2 & 1.0 & $\mathrm{R}$ mass & 4 & 0.6 & No & L, no breast & Fibroadenoma & US core biopsy \\
\hline 7 & 1 & 2.7 & $\mathrm{R}$ mass & 6 & 7.2 & No & $\mathrm{L}, 1.1$ & $\begin{array}{l}\text { IDC, poorly } \\
\text { differentiated }\end{array}$ & $\begin{array}{l}\text { Mastectomy (normal } \\
\text { L prophylactic } \\
\text { mastectomy) }\end{array}$ \\
\hline 7 & 2 & 4.3 & $\mathrm{R}$ mass & 6 & 12 & No & $L, 1.1$ & $\begin{array}{l}\text { IDC, poorly } \\
\text { differentiated }\end{array}$ & Mastectomy \\
\hline 7 & 3 & 1.3 & $\mathrm{R}$ mass & 5 & 4.5 & No & $L, 1.1$ & $\begin{array}{l}\text { IDC, poorly } \\
\text { differentiated }\end{array}$ & Mastectomy \\
\hline 8 & 1 & 1.5 & L mass & 6 & 0.9 & No & $\mathrm{R}, 0.6$ & $\begin{array}{l}\text { DCIS, poorly } \\
\text { differentiated }\end{array}$ & Mastectomy \\
\hline 8 & 2 & 1.0 & L nonmass & 3 & 1.3 & No & $\mathrm{R}, 1.1$ & Fibrocystic change & Mastectomy \\
\hline 8 & 3 & 1.4 & R nonmass & 4 & 0.8 & Yes & L, 0.6 & Fibrocystic change & Surgical excision \\
\hline 9 & 1 & 1.0 & L mass & 4 & 0.7 & No & $R, 0.8$ & Radial scar & Surgical excision \\
\hline 10 & 1 & 5.2 & L mass & 6 & 4.6 & No & $R, 0 . .4$ & $\begin{array}{l}\text { IDC, poorly } \\
\text { differentiated }\end{array}$ & Mastectomy \\
\hline 11 & 1 & 2.0 & $\mathrm{R}$ mass & 6 & 2.98 & No & $\mathrm{L}, 1.17$ & $\begin{array}{l}\text { IDC, poorly } \\
\text { differentiated }\end{array}$ & Lumpectomy \\
\hline 11 & 2 & 0.9 & $\mathrm{~L}$ mass & 3 & 1.27 & Yes & $\mathrm{R}, 1.27$ & $\mathrm{~N} / \mathrm{A}$ & $17 \mathrm{mo}$ \\
\hline 12 & 1 & 2.2 & L mass & 6 & 5.94 & No & $R, 0.92$ & $\begin{array}{l}\text { IDC, moderately } \\
\text { differentiated }\end{array}$ & Lumpectomy \\
\hline 12 & 2 & 0.7 & $\mathrm{~L}$ mass & 3 & 0.26 & No & $R, 0.25$ & $\mathrm{~N} / \mathrm{A}$ & $12 \mathrm{mo}$ \\
\hline 12 & 3 & 0.6 & L mass & 3 & 0.33 & No & $\mathrm{R}, 0.7$ & $\mathrm{~N} / \mathrm{A}$ & $12 \mathrm{mo}$ \\
\hline 13 & 1 & 1.4 & L mass & 6 & 1.17 & No & $\mathrm{R}, 1.1$ & $\begin{array}{l}\text { IDC \& ILC moderately } \\
\text { differentiated }\end{array}$ & Lumpectomy \\
\hline 13 & 2 & 1.2 & R nonmass & 4 & 0.63 & Yes & L, 0.64 & N/A & $20 \mathrm{mo}$ \\
\hline 14 & 1 & 4.0 & L nonmass & 4 & 3.2 & No & $\mathrm{R}, 1.03$ & $\begin{array}{l}\text { IDC \& ILC moderately } \\
\text { differentiated }\end{array}$ & Mastectomy \\
\hline 15 & 1 & 1.0 & $\mathrm{~L}$ mass & 3 & 0.8 & Yes & $R, 0.9$ & N/A & $13 \mathrm{mo}$ \\
\hline 15 & 2 & 0.7 & $\mathrm{R}$ mass & 3 & 0.7 & No & $L, 0.6$ & Lymph node & FNA biopsy \\
\hline 16 & 1 & 4.0 & L mass & 6 & 9.2 & No & $\mathrm{R}, 0.8$ & $\begin{array}{l}\text { IDC, moderately } \\
\text { differentiated }\end{array}$ & Mastectomy \\
\hline 16 & 2 & 9.0 & L nonmass & 4 & 1.85 & No & $\mathrm{R}, 1.1$ & DCIS, high grade & Mastectomy \\
\hline 17 & 1 & 3.4 & L mass & 6 & 5.58 & No & $\mathrm{R}, 0.9$ & $\begin{array}{l}\text { IDC, moderately } \\
\text { differentiated }\end{array}$ & Mastectomy \\
\hline 17 & 2 & 1.6 & L mass & 6 & 5.8 & No & $R, 0.9$ & $\begin{array}{l}\text { IDC, moderately } \\
\text { differentiated }\end{array}$ & Mastectomy \\
\hline 17 & 3 & 9.0 & L nonmass & 5 & 0.4 & No & $R, 0.85$ & DCIS, high grade & Mastectomy \\
\hline 18 & 1 & 2.0 & $\mathrm{R}$ mass & 3 & 1.1 & No & $L, 1.2$ & Seroma cavity & Mastectomy \\
\hline 18 & 2 & 9.0 & $\mathrm{R}$ nonmass & 3 & 0.8 & No & $L, 0.5$ & DCIS, high grade & Mastectomy \\
\hline 18 & 3 & 2.0 & L mass & 3 & 1.64 & Yes & $R, 0.5$ & Seroma cavity & $\begin{array}{l}\text { 13-mo PET } \\
\text { 6/16/06, negative }\end{array}$ \\
\hline 19 & 1 & 1.7 & $\mathrm{~L}$ mass & 6 & 4.0 & No & $\mathrm{R}, 0.7$ & $\begin{array}{l}\text { IDC, moderately } \\
\text { differentiated }\end{array}$ & Mastectomy \\
\hline 19 & 2 & 1.1 & $\mathrm{~L}$ mass & 4 & 0.6 & No & $\mathrm{R}, 0.6$ & $\begin{array}{l}\text { IDC, moderately } \\
\text { differentiated }\end{array}$ & Mastectomy \\
\hline 19 & 3 & 1.1 & L mass & 4 & 0.6 & No & $\mathrm{R}, 0.7$ & $\begin{array}{l}\text { IDC, moderately } \\
\text { differentiated }\end{array}$ & Mastectomy \\
\hline 19 & 4 & 1.0 & L mass & 4 & 0.4 & No & $\mathrm{R}, 0.4$ & $\begin{array}{l}\text { IDC, moderately } \\
\text { differentiated }\end{array}$ & Mastectomy \\
\hline
\end{tabular}


TABLE 3

(Continued)

\begin{tabular}{|c|c|c|c|c|c|c|c|c|c|}
\hline Patient & Lesion & $\begin{array}{l}\text { Size } \\
(\mathrm{cm})\end{array}$ & $\begin{array}{c}\text { MRI } \\
\text { finding* }\end{array}$ & $\begin{array}{c}\text { BIRADS } \\
\text { assessment }\end{array}$ & $\begin{array}{l}\text { PET/CT } \\
\text { finding } \\
\text { maximum } \\
\text { SUV }\end{array}$ & $\begin{array}{c}\text { Contralateral } \\
\text { breast }^{\dagger}\end{array}$ & $\begin{array}{c}\text { Normal SUV } \\
\text { contralateral } \\
\text { breast }^{\ddagger}\end{array}$ & Pathology & $\begin{array}{l}\text { Follow-up } \\
\text { or histology }\end{array}$ \\
\hline 19 & 5 & 0.9 & $\mathrm{~L}$ mass & 4 & 0.6 & No & L, 0.4 & $\begin{array}{l}\text { IDC, moderately } \\
\text { differentiated }\end{array}$ & Mastectomy \\
\hline 19 & 6 & 1.5 & $\mathrm{~L}$ mass & 3 & 0.6 & Yes & $\mathrm{R}, 0.9$ & Fibrocystic changes & Surgical excision \\
\hline 20 & 1 & 1.6 & R nonmass & $1 \S$ & 1.3 & No & L, 0.8 & DCIS, high grade & Mastectomy \\
\hline 21 & 1 & 10.0 & L nonmass & 5 & 3.3 & No & $\mathrm{R}, 0.7$ & $\begin{array}{l}\text { IDC, poorly } \\
\text { differentiated }\end{array}$ & Mastectomy \\
\hline 21 & 2 & 2.0 & $L$ mass & 6 & 3.2 & No & $\mathrm{R}, 0.7$ & DCIS, high grade & Mastectomy \\
\hline 22 & 1 & 2.2 & $\mathrm{~L}$ mass & 6 & 1.7 & No & $R, 0.28$ & DCIS, high grade & Mastectomy \\
\hline 22 & 2 & 0.9 & $\mathrm{R}$ nonmass & 4 & 1.1 & Yes & L, 0.9 & Fibrocystic tissue & Mastectomy \\
\hline \multicolumn{10}{|c|}{ 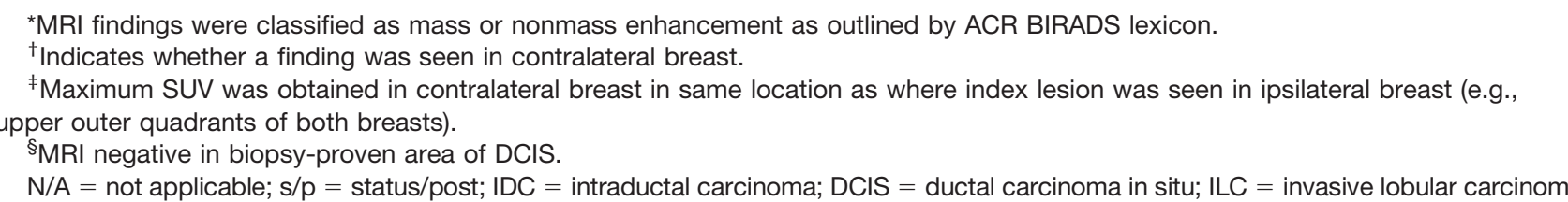 } \\
\hline
\end{tabular}

presented with an incidental lesion in each breast as detected on MRI. Figure 2 shows orthogonal slices at the level of a 1-cm round, homogeneously enhancing MRIdetected mass, characterized as BIRADS 3 (probably benign). Dedicated mammography and a second-look US revealed no suspicious abnormality. No increased uptake was seen on the PET scan. After 17 mo of follow-up, the patient remains disease free.

Patient 2, a 44-y-old woman, presented with 3 masses in the left breast. Figure 3 shows orthogonal slices at the level of a 2.2-cm irregular, heterogeneously enhancing mass detected on both the MRI and PET scans. This mass was confirmed by pathology to be moderately differentiated intraductal carcinoma (IDC). Figure 4 shows a 3D rendition with the MRI breasts semitransparent and the PET lesion superimposed. Figure 5 shows orthogonal slices at the level of a 0.6-cm enhancing mass detected only on the MRI scan.

TABLE 4

Results for MRI vs. Fused MRI/PET (45 Lesions)

\begin{tabular}{lcc}
\hline \multicolumn{1}{c}{ Parameter } & MRI & MRI/PET \\
\hline TN* $^{*}(n)$ & $11(24.4)$ & $20(44.4)$ \\
$\mathrm{TP}^{*}(n)$ & $22(48.8)$ & $15(33.3)$ \\
$\mathrm{FN}^{*}(n)$ & $2(4.4)$ & $9(20)$ \\
$\mathrm{FP}^{*}(n)$ & $10(22.2)$ & $1(2.2)$ \\
Total $^{*}(n)$ & $45(99.8)$ & $45(99.9)$ \\
Sensitivity (\%) & 92 & 63 \\
Specificity (\%) & 52 & 95 \\
PPV (\%) & 69 & 94 \\
NPV (\%) & 85 & 69 \\
& & \\
\hline & & \\
\hline
\end{tabular}

This mass was correctly categorized as BIRADS 3 (probably benign) and exhibited no uptake on the PET scan.

Patient 3, a 27-y-old woman, presented with 1 enhancing mass in each breast. Figure 6 shows orthogonal slices, at the level of a 2.2-cm irregular, heterogeneous mass detected in the left breast on both the MRI and PET scans. The PET study demonstrated a focus of uptake with a SUV of 1.7. At mastectomy, the mass in the left breast was found to be high-grade ductal carcinoma in situ (DCIS). The patient underwent a right mastectomy, revealing a benign mass as predicted by the negative PET scan.

\section{Role of PET in Evaluation of Suspicious Lesions}

Fourteen of 45 lesions $(31.1 \%)$ were categorized as BIRADS 6, known cancer. The mean size of the lesions was $2.7 \mathrm{~cm}$ (range, $1.4-5.2 \mathrm{~cm}$ ). The SUVs of these lesions averaged 5.04 (range, 0.9-12), whereas the same region in the contralateral breast averaged 0.65 (range, 0.4-1.17). PET correctly identified all lesions as a focus of increased activity.

Because the role of ${ }^{18} \mathrm{~F}-\mathrm{FDG}$ PET may be limited in the detection of small $(<1 \mathrm{~cm})$ primary breast lesions, all enhancing masses that were $1 \mathrm{~cm}$ or greater and categorized as BIRADS 4 and $5(12 / 45 \%-26.6 \%)$ were reevaluated. The mean lesion size was $2.6 \mathrm{~cm}$ (range, $1.0-9.0 \mathrm{~cm}$ ), and the SUVs averaged 1.47 (range, 0.4-4.5), whereas the same region in the contralateral breast averaged 0.68 (range, $0.4-$ 1.1). PET identified 4 of $8(50 \%)$ malignancies correctly as a focus of increased activity. Three biopsy proven-benign lesions and 1 lesion being followed radiographically (4/4) were correctly not seen on PET.

We then reevaluated all enhancing masses (10/45, 22.2\%) that were $1 \mathrm{~cm}$ or greater and categorized as BIRADS 3 . The mean lesion size was $2.5 \mathrm{~cm}$ (range, $1.0-9.0 \mathrm{~cm}$ ) and the 

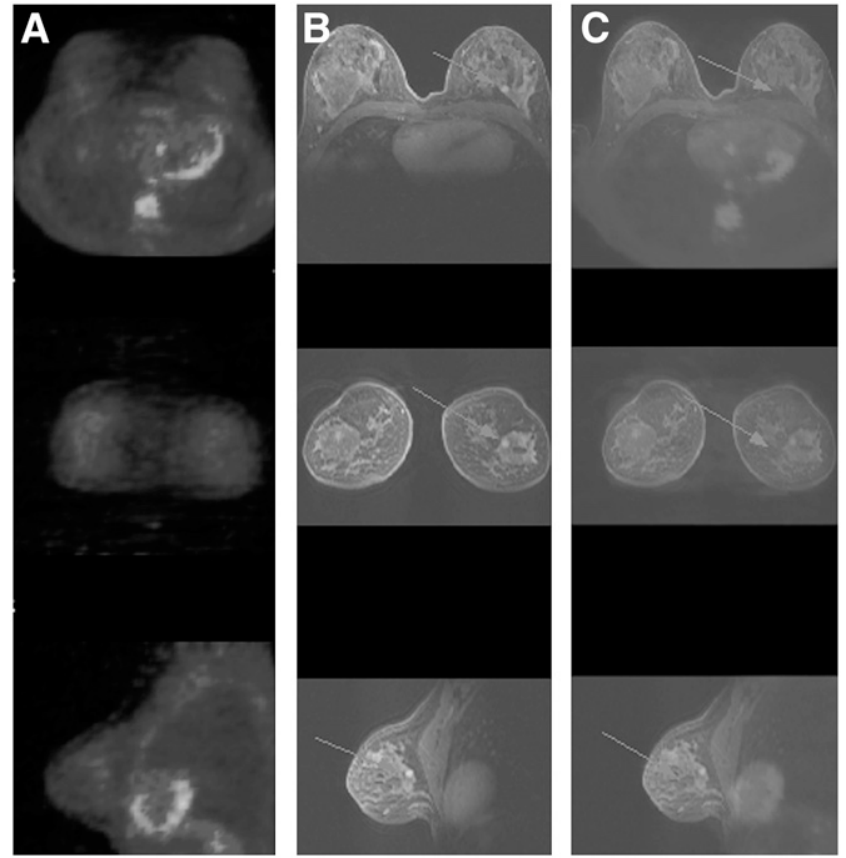

FIGURE 2. Patient 1, a 25-y-old woman, presented with 1 mass in each breast. Axial (top), coronal (middle), and sagittal (bottom) slices are shown at level of $1-\mathrm{cm}$ enhancing mass (arrows) detected only on MRI scan, evaluated as a probably benign finding and found to be benign after 17 mo of follow-up. (A) Unregistered PET scan. (B) Original MRI scan. (C) Registered PET scan superimposed on original MRI scan. No increased uptake was seen on PET scan.

SUVs averaged 1.22 (range, 0.6-1.82), whereas the same region in the contralateral breast averaged 0.63 (range, $0.17-$ 1.27). PET identified foci of activity in 3 lesions. Six negative lesions are being followed radiographically. For the other 4 lesions, 2 were negative and 1 was positive (DCIS) on mastectomy, and 1 was negative after surgical excision.

In all known cancers, 12 of 24 had foci of uptake with an SUV of 3.0 or greater and do not fall in the overlap region between negative and positive lesions.

\section{DISCUSSION}

The principal applications of ${ }^{18} \mathrm{~F}-\mathrm{FDG}$ PET in breast cancer are in disease restaging and treatment monitoring (16-19). However, ${ }^{18} \mathrm{~F}-\mathrm{FDG}$ PET could be indicated for preoperative staging and primary diagnosis $(17,18,20-24)$. Breast cancer detection is still principally based on mammography, which misses about $10 \%$ of breast carcinomas (25-28) because of dense breast tissue. Breast MRI has been shown to be highly effective in identifying occult tumor, but FP findings may occur (6,29-34). As both MRI and PET/CT are frequently requested for disease evaluation for suspected breast cancer, a mechanism to foster direct comparison of lesions detected with both modalities would be useful (7-9).

\section{Problems with Supine PET}

The biggest limitation in comparing prone MR images with supine PET images is the loss of normal landmarks.
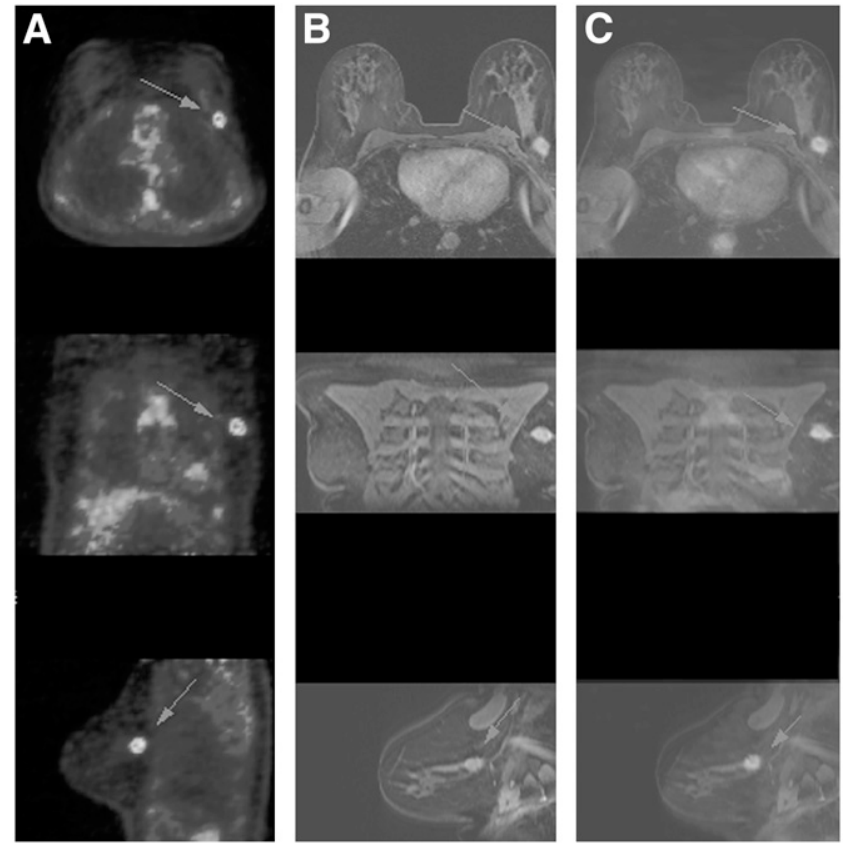

FIGURE 3. Patient 2, a 44-y-old woman, presented with 3 masses in left breast. Axial (top), coronal (middle), and sagittal (bottom) slices are shown at level of $2-\mathrm{cm}$ enhancing mass (arrows) detected on both MRI and PET scans and confirmed by pathology to be moderately differentiated IDC. (A) Unregistered PET scan. (B) Original MRI scan. (C) Registered PET scan superimposed on original MRI scan.

Marked differences in patient positioning result in unreliability of the usual landmarks - the quadrant of the breast where an abnormality is located and the distance of the lesion from the nipple. Superimposing PET and MR images may increase the specificity of breast MRI, but only if the breasts are in the same position - that is, prone. The breasts become markedly distorted when supine/prone images are registered and it is challenging to identify the corresponding lesion in all 3 orthogonal planes of the fused images (35). Several groups have suggested that an independent PET scan should be acquired in the prone position to increase the quality of PET in the breast $(7,8,36-40)$.

\section{Prone MRI and PET}

Goerres et al. (8) compared side-by-side prone ${ }^{18} \mathrm{~F}$-FDG PET and MRI studies in 32 patients with findings suspicious for locoregional recurrence, chest wall recurrence, or a secondary tumor on the contralateral side. They addressed the issue of prone PET by having the PET scan performed in a position as identical to the MRI as possible by using a conventional MRI breast coil holder with the receiver coil removed. Sensitivity, specificity, and accuracy were $79 \%$, $94 \%$, and $88 \%$ for MRI, respectively, compared with $100 \%$, $72 \%$, and $84 \%$ for ${ }^{18} \mathrm{~F}-\mathrm{FDG}$ PET, respectively. These numbers were based on the entire breast, and thoracic wall involvement, rather than on a lesion-by-lesion basis.

For prone imaging evaluation the technologist attempted to use the same patient positioning (angle of the chest and 

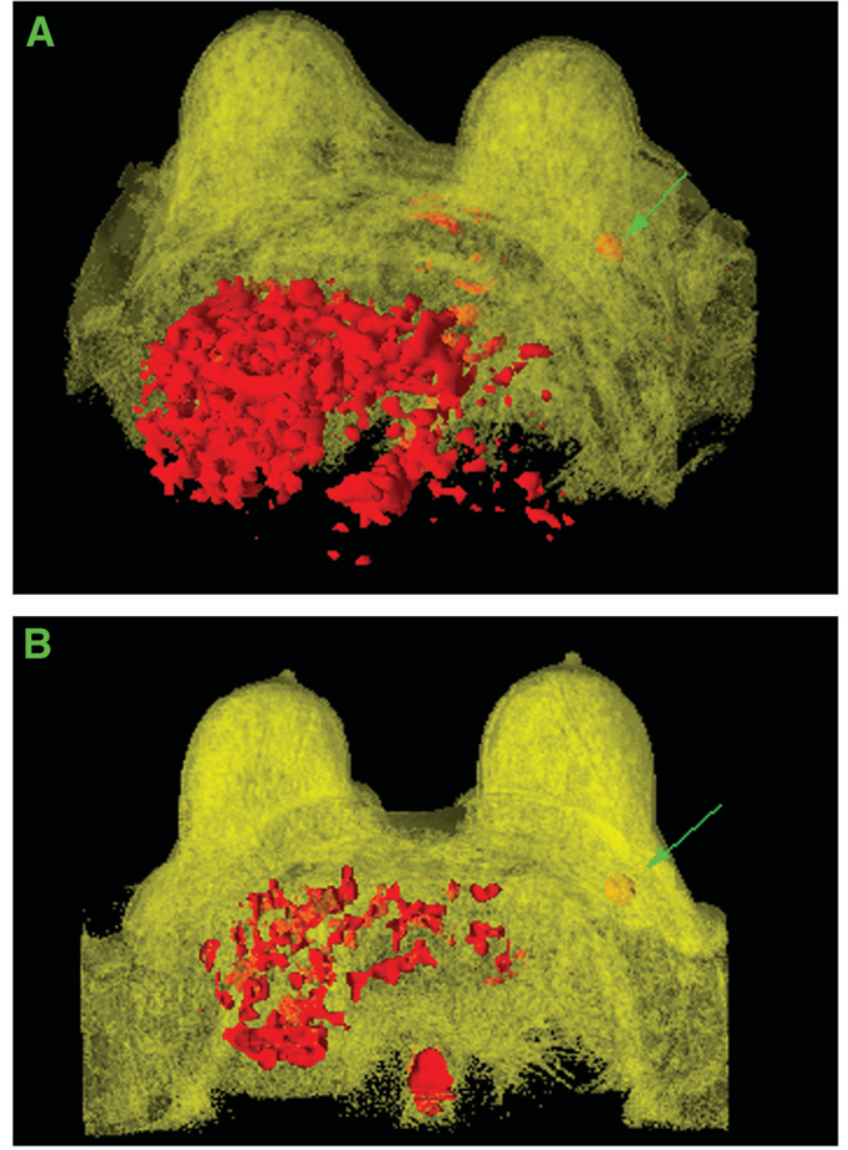

FIGURE 4. Patient 2 shown in 3D with arrow indicating 2-cm lesion confirmed as moderately differentiated IDC. Yellow, shown transparent, is MRI scan with fused PET lesion and other high-activity regions (liver and spine) superimposed in red. Lesion is inside left breast, whereas liver and spine are in front of and beneath MRI scan. (A) 3D view from 1 angle with level of PET activity set at 1 particular level. (B) 3D view from a different angle with level of PET activity set at a higher level than in A.

abdomen to the breast, maintaining an identical position for the patient's arms, and positioning of the breast) in PET as was used in MRI, to minimize the chance of a mismatch of the superimposed images. However, position and shape changes in the breast between the MRI and PET occurred for all patients, especially in women with large breasts. Fortunately, the fusion technique can correct for differences in the patient positioning. Several PET breast apparati with different cup sizes to ensure that the shape of the breast is maintained are planned.

Coregistration of MRI and PET images using semiautomated fusion software increased both readers' confidence in characterizing a lesion as benign or malignant in 17 of the 22 cases. In all 7 patients with equivocal MRI findings in the contralateral breast, PET correctly did not detect a corresponding focus of activity.

On PET, normal tissue SUVs can be calculated to help increase the certainty of the viewer, but semiquantitative analysis alone cannot identify malignant lesions with high specificity in breast tissue. When there is an area of increased uptake, the reader must rely on experience to determine the
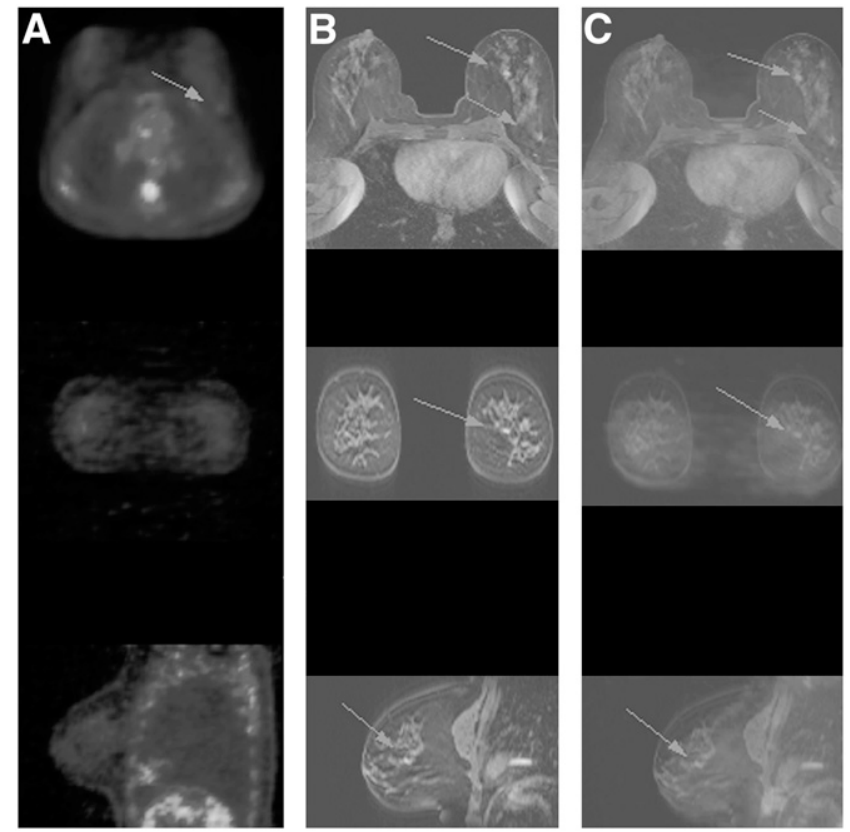

FIGURE 5. Patient 2 shown with axial (top), coronal (middle), and sagittal (bottom) slices now at level of a $0.6-\mathrm{cm}$ enhancing mass (upper arrows) detected only on MRI scan and evaluated as a BIRADS 3, probably benign finding. (A) Unregistered PET scan. (B) Original MRI scan. (C) Registered PET scan superimposed on original MRI scan. The $2-\mathrm{cm}$ mass shown in Figure 3 is just visible in bottom right of axial view (lower arrow).

malignant potential of a lesion. To our knowledge, there is no definitive threshold SUV to distinguish between benign and malignant lesions in the breast. As expected, in our small patient population, there was a significant overlap of SUV range for both benign and malignant lesions. The size limitation of a lesion that may be detected by PET/CT is also unknown. In this study, abnormal metabolic uptake was consistently seen in malignancies greater than $1 \mathrm{~cm}, 15$ of $22(68.2 \%)$ lesions.

\section{Management of Breast Lesions}

Our preliminary results suggest that prone PET breast imaging combined via a fusion technique facilitates a better correlation of lesions. A positive lesion on PET and MRI increases the certainty of malignancy (PPV) and may convince patients that they should undergo a biopsy. One concern with these results is that although specificity is increased, it is at the cost of sensitivity. The low sensitivity (63\%) and NPP (69\%) of the fusion technique do not allow a referring physician to be certain that the indeterminate lesion on MRI is truly benign. We penalized PET (FN) when separate MRI lesions that were small and close together were not distinguished as separate lesions on PET (Table 3). Had we not done so, the sensitivity and PPV of the MRI and PET results would have been increased to $83 \%$ and $82 \%$, respectively.

\section{Limitations}

In our population, PET did not consistently show increased activity at sites of DCIS. PET did identity as 

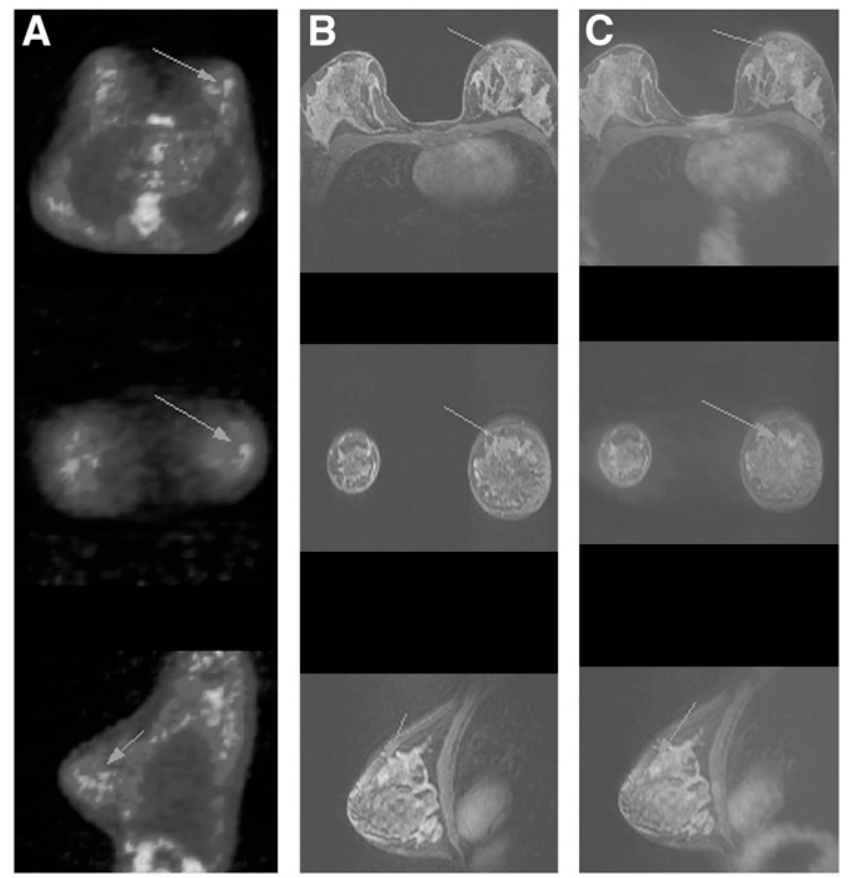

FIGURE 6. Patient 3 , a 27-y-old woman, presented with 1 enhancing mass in each breast. Axial (top), coronal (middle), and sagittal (bottom) slices are shown at level of a 2.2-cm mass detected in left breast (arrows) on both MRI and PET scans and found to be poorly differentiated DCIS at mastectomy. (A) Unregistered PET scan. (B) Original MRI scan. (C) Registered PET scan superimposed on original MRI scan. As can be seen in $A$ on axial and coronal views, mass in right breast was correctly not seen on PET scan as determined also by mastectomy.

hypermetabolic all of the cancers that were IDC. Additional work is needed to determine the potential of PET in both DCIS and invasive lobular carcinoma.

\section{CONCLUSION}

Acquisition of prone PET scans using the newly designed positioning device permitted acquisition of prone scans suitable for fusion with breast MRI scans. The functional tumor information provided by ${ }^{18} \mathrm{~F}$-FDG PET, combined with the anatomic localization provided by MRI, yields an improved diagnostic tool for both primary and recurrent breast cancer. Fused PET and MRI scans substantially increased the specificity of MRI but decreased the sensitivity in this small group of patients. Additional data are needed to statistically confirm the hypothesis that the additional information provided by fused PET and MR images potentially helps in the evaluation of enhancing lesions detected on breast MRI.

\section{ACKNOWLEDGMENTS}

We thank the referees for their suggestions. This work was partially supported by a New York University Department of Radiology Seed grant and was presented in part at the 53rd Annual Meeting of the Society of Nuclear Medicine, San Diego, California, June 3-7, 2006.

\section{REFERENCES}

1. Stoutjesdijk MJ, Boetes C, Jager GJ, et al. Magnetic resonance imaging and mammography in women with a hereditary risk of breast cancer. J Natl Cancer Inst. 2001;93:1095-1102.

2. Warner E, Plewes DB, Shumak RS, et al. Comparison of breast MRI, mammography, and ultrasound for surveillance of women at high risk for hereditary breast cancer. J Clin Oncol. 2001;19:3524-3531.

3. Wright H, Listinsky J, Rim A, et al. Magnetic resonance imaging as a diagnostic tool for breast cancer in premenopausal women. Am J Surg. 2005;190: 572-575.

4. Teifke A, Hlawatsch A, Beier T, et al. Undetected malignancies of the breast: dynamic contrast-enhanced MR imaging at 1.0 T. Radiology. 2002;224:881-888.

5. Liberman L, Morris EA, Lee MJ, et al. Breast lesions detected on MR imaging: features and positive predictive value. AJR. 2002;179:171-178.

6. Hathaway PB, Mankoff DA, Maravilla KR, et al. Value of combined FDG PET and MR imaging in the evaluation of suspected recurrent local-regional breast cancer: preliminary experience. Radiology. 1999;210:807-814.

7. Yutani K, Tatsumi M, Uehara T, Nishimura T. Effect of patients' being prone during FDG PET for the diagnosis of breast cancer. AJR. 1999;173:13371339 .

8. Goerres G, Michel S, Fehr M, et al. Follow-up of women with breast cancer: comparison between MRI and FDG PET. Eur Radiol. 2003;13:1635-1644.

9. Tatsumi M, Cohade C, Mourtzikos KA, Fishman EK, Wahl RL. Initial experience with FDG-PET/CT in the evaluation of breast cancer. Eur J Nucl Med Mol Imaging. 2006;33:254-262.

10. Pichler BJ, Judenhofer MS, Catana C, et al. Performance test of an LSO-APD detector in a 7-T MRI scanner for simultaneous PET/MRI. J Nucl Med. 2006; 47:639-647.

11. Moy L, Noz ME, Maguire GQ Jr, et al. Prone mammoPET acquisition improves the ability to fuse MRI and PET breast scans. Clin Nucl Med. 2007;32:194-198.

12. Pfluger T, Vollmar C, Wismuller A, et al., Quantitative comparison of automatic and interactive methods for MRI-SPECT image registration of the brain based on 3-dimensional calculation of error. J Nucl Med. 2000;41:1823-1829.

13. Noz ME, Maguire GQ Jr, Zeleznik MP, Kramer EL, Mahmoud F, Crafoord J. A versatile functional-anatomic image fusion method for volume data sets. $J$ Med Syst. 2001;25:297-307.

14. Olivecrona L, Crafoord J, Olivecrona H, et al. Acetabular component migration in total hip arthroplasty using CT and a semi-automated program for volume merging. Acta Radiol. 2002;43:517-527.

15. Gorniak RJT, Kramer EL, Maguire GQ Jr, Noz ME, Schettino CJ, Zeleznik MP. Evaluation of a semi-automatic 3D fusion technique applied to molecular imaging and MRI brain/frame volume data sets. J Med Syst. 2003;27:141-156.

16. Biersack HJ, Palmedo H. Locally advanced breast cancer: Is PET useful for monitoring primary chemotherapy? J Nucl Med. 2003;44:1815-1817.

17. Avril N, Rose CA, Schelling M, et al. Breast imaging positron emission tomography and fluorine-18 fluorodeoxyglucose: use and limitations. J Clin Oncol. 2000;18:3495-3502.

18. Bombardieri E, Aktolun C, Baum RP, et al. FDG-PET: procedure guidelines for tumor imaging. Eur J Nucl Med Mol Imaging. 2003;30:BP115-BP124.

19. Leung JWT. New modalities in breast imaging: digital mammography, positron emission tomography, and sestamibi scintimammography. Radiol Clin North Am. 2002;40:467-482.

20. Bombardieri E, Crippa F, Baio SM, Peeters BA, Greco M, Pauwels EK. Nuclear medicine advances in breast cancer imaging. Tumori. 2001;8:277-287.

21. Minn H, Soini I. $\left[{ }^{18} \mathrm{~F}\right]$ Fluorodeoxyglucose scintigraphy in diagnosis and follow up of treatment in advanced breast cancer. Am J Clin Pathol. 1989;91:535-541.

22. Kubota K, Matsuzawa T, Amemiya A, et al. Imaging of breast cancer with F-18 fluorodeoxyglucose and positron emission tomography. J Comput Assist Tomogr. 1989;13:1097-1098.

23. Avril N, Schelling M, Dose J, et al. Utility of PET in breast cancer. Clin Positron Imaging. 1999;2:261-271.

24. Moon DH, Maddahi J, Silverman DH, Glaspy JA, Phelps ME, Hoh CK. Accuracy of whole-body fluorine-18-FDG PET for the detection of recurrent or metastatic breast carcinoma. J Nucl Med. 1998;39:431-435.

25. Fletcher SW, Black W, Harris R. Report of international workshop on screening for breast cancer. J Natl Cancer Inst. 1993;85:1644-1656.

26. Tabar L, Duffy SW, Krusemo UB. Detection method, tumor size and node metastasis in breast cancer diagnosed during a trial of breast cancer screening. Eur J Cancer Clin Oncol. 1987;23:959-962.

27. Kopans DB. The positive predictive value of mammography. AJR. 1992;158: 521-526.

28. Bird RE, Wallace TW, Yankaskas BC. Analysis of cancer missed at screening mammography. Radiology. 1992;184:613-617. 
29. Buchanan CL, Morris EA, Dorn PL, Borgen PI, Van Zee KJ. Utility of breast magnetic resonance imaging in patients with occult primary breast cancer. Ann Surg Oncol. 2005;12:1045-1053.

30. Groves AM, Warren RM, Godward S, Rajan PS. Characterization of pure highgrade DCIS on magnetic resonance imaging using the evolving breast MR lexicon terminology: Can it be differentiated from pure invasive disease? Magn Reson Imaging. 2005;23:733-738.

31. Malich A, Fischer DR, Wurdinger S, et al. Potential MRI interpretation model: differentiation of benign from malignant breast masses. AJR. 2005;185: 964-970.

32. Lehman CD, Blume JD, Weatherall P, et al. International Breast MRI Consortium Working Group: screening women at high risk for breast cancer with mammography and magnetic resonance imaging. Cancer. 2005;103:1898-1905.

33. Mumtaz H, Davidson T, Hall-Craggs MA, et al. Comparison of magnetic resonance imaging and conventional triple assessment in locally recurrent breast cancer. Br J Surg. 1997;84:1147-1151.

34. Gilles R, Guinebretiere JM, Shapeero LG, et al. Assessment of breast cancer recurrence with contrast-enhanced subtraction MR imaging: preliminary results in 26 patients. Radiology. 1993;188:473-478.
35. Pietrzyk U, Scheidhauer K, Scharl A, Schuster A, Schicha H. Presurgical visualization of primary breast carcinoma with PET emission and transmission imaging. J Nucl Med. 1995;36:1882-1884.

36. Avril N, Scheidhauer K, Kuhn W. Dual-modality PET/CT imaging for clinical oncology using a single tomograph. In: Wieler HJ, Coleman RE, eds. PET in Clinical Oncology. Darmstadt, Germany: Springer; 2000:355-371.

37. Dose J, Bleckmann C, Bachmann S, et al. Comparison of fluorodeoxyglucose positron emission tomography and "conventional diagnostic procedures" for the detection of distant metastases in breast cancer patients. Nucl Med Commun. 2002; 23:857-864.

38. Krak NC, van der Hoeven JM, Hoekstra OS, et al. Measuring $\left[{ }^{18}\right.$ F $]$ FDG uptake in breast cancer during chemotherapy: comparison of analytical methods. Eur J Nucl Med Mol Imaging. 2003;30:674-681.

39. Brix G, Henze M, Knopp N, et al. Comparison of pharmacokinetic MRI and $\left[{ }^{18} \mathrm{~F}\right]$ fluorodeoxyglucose PET in the diagnosis of breast cancer: initial experience. Eur Radiol. 2001;11:2058-2070.

40. Landheer ML, Steffens MG, Klinkenbijl JH, Westenberg AH, Oyen WJ. Value of fluorodeoxyglucose positron emission tomography in women with breast cancer. Br J Surg. 2005;92:1363-1367. 